\title{
Acyl Ghrelin Improves Synapse Recovery in an In Vitro Model of Postanoxic Encephalopathy
}

\author{
Irina I. Stoyanova ${ }^{1}$ - Jeannette Hofmeijer ${ }^{1,3}$ - Michel J. A. M. van Putten ${ }^{1,4}$ • \\ Joost le Feber ${ }^{1,2}$
}

Received: 8 August 2015 / Accepted: 19 October 2015 / Published online: 6 November 2015

(C) The Author(s) 2015. This article is published with open access at Springerlink.com

\begin{abstract}
Comatose patients after cardiac arrest have a poor prognosis. Approximately half never awakes as a result of severe diffuse postanoxic encephalopathy. Several neuroprotective agents have been tested, however without significant effect. In the present study, we used cultured neuronal networks as a model system to study the general synaptic damage caused by temporary severe hypoxia and the possibility to restrict it by ghrelin treatment. Briefly, we applied hypoxia $\left(\mathrm{pO}_{2}\right.$ lowered from 150 to $20 \mathrm{mmHg}$ ) during $6 \mathrm{~h}$ in $55 \mathrm{cul}-$ tures. Three hours after restoration of normoxia, half of the cultures were treated with ghrelin for $24 \mathrm{~h}$, while the other, non-supplemented, were used as a control. All cultures were processed immunocytochemically for detection of the synaptic marker synaptophysin. We observed that hypoxia led to drastic decline of the number of synapses, followed by some recovery after return to normoxia, but still below the prehypoxic level. Additionally, synaptic vulnerability was selective: large- and small-sized neurons were more susceptible to synaptic damage than the medium-sized ones. Ghrelin treatment significantly increased the synapse density, as compared with the non-treated controls or with the prehypoxic period.
\end{abstract}

Irina I. Stoyanova

stoyanovai@yahoo.co.uk

1 Department of Clinical Neurophysiology, Faculty of Science and Technology, University of Twente, Building Carré 3714, P.O. Box 217, 7500 AE Enschede, The Netherlands

2 Department of Biomedical Signals and Systems, EWI, University of Twente, Enschede, The Netherlands

3 Department of Neurology, Rijnstate Hospital, Arnhem, The Netherlands

4 Department of Clinical Neurophysiology, Medisch Spectrum Twente, Enschede, The Netherlands
The effect was detected in all neuronal subtypes. In conclusion, exogenous ghrelin has a robust impact on the recovery of cortical synapses after hypoxia. It raises the possibility that ghrelin or its analogs may have a therapeutic potential for treatment of postanoxic encephalopathy.

Keywords Brain hypoxia - Postanoxic encephalopathy · Synapse density $\cdot$ Ghrelin

\section{Introduction}

Comatose patients after cardiac arrest have a poor prognosis. Approximately half never awakes as a result of severe diffuse postanoxic encephalopathy [1]. Several neuroprotective agents such as barbiturates [2], calcium channel blockers [3, 4], magnesium [5], and diazepam [6] have been tested, however without significant effect. The only treatment of proven benefit is mild therapeutic hypothermia [7,8], although the gain of this treatment has become uncertain since the recent Targeted Temperature Management (TTM) trial [9]. An important rationale behind all neuroprotective strategies, including hypothermia, has been prevention of secondary damage by inhibition of activation. This should preserve remaining energy levels in order to maintain membrane voltage and basic cellular processes and prevent excitotoxicity [10]. In acute ischemic stroke, where part of the same mechanisms play a role, many modalities have been tested based on the same argumentation [11]. Neither in postanoxic coma nor in ischemic stroke were any of these beneficial in clinical trials.

The initial encephalopathy after cardiac arrest results from a global decrease of cerebral perfusion to a level that is insufficient to meet the brain's high metabolic demands [12]. Classically, the resulting ATP depletion is associated with failure of transmembrane ion pumps, depolarization, cell swelling, 
and cell death [13]. However, in imaging studies, sings of cell swelling and necrosis were only moderately associated with severity of encephalopathy and even absent in almost half of patients, despite lasting severe encephalopathy and a poor outcome [14]. Other pathophysiological mechanisms leading to brain malfunctioning include local acidosis, free radical formation, and nitric oxide production. Functional changes are caused by damage to mitochondria, cytoskeleton, and glutamate receptors $[15,16]$.

In animal models of acute ischemic stroke, synaptic failure through impaired transmitter release was an early consequence of ischemia and led to irreversible cerebral network damage in the absence of cell swelling or necrosis [17]. Synaptic transmission disturbances cause a lack of postsynaptic activation and consequently decreased network activity [18]. Since long-term suppression of activity leads to permanent damage $[19,20]$ and lack of brain activity during more than $24 \mathrm{~h}$ is strongly associated with absence of recovery from postanoxic coma [21,22], it is argued that mild excitation such as caused by ghrelin, instead of inhibition, may be of benefit.

Previously, we have shown improvements of network connectivity along with an increased number of synapses, resulting from chronic stimulation of dissociated cortical neurons with ghrelin $[23,24]$. Ghrelin is multifunctional 28aminoacid hormone and a neuropeptide originally not only found in the rat stomach [25], but also identified in the hypothalamus, to a lesser extent in the hippocampus and brain cortex [26-28], as well as in the adipose tissue [29]. Ghrelin gene encodes a precursor which is cleaved to produce first unacylated ghrelin (des-acyl ghrelin (DAG)) further transformed into acyl ghrelin (AG, also referred to as ghrelin) by esterification of the serine- 3 residue with $n$-octanoic acid [30].
AG binds to the growth hormone secretagogue receptor 1a isoform (GHSR1) [25], which is expressed in the cerebral cortex in vivo [27], as well as in dissociated cortical neurons [23], and is responsible for some of the central and peripheral effects of ghrelin [31]. As recently reported, ghrelin enhances synaptic plasticity [26], memory consolidation [32], and adult neurogenesis $[33,34]$. Here, we show that ghrelin improves synaptic recovery in an in vitro model of postanoxic encephalopathy consisting of cultured neuronal networks exposed to severe hypoxia.

\section{Material and Methods}

We used an in vitro model of postanoxic encephalopathy consisting of cultured neuronal networks exposed to severe hypoxia for $6 \mathrm{~h}$, followed by a recovery period with normoxia during $3 \mathrm{~h}$. Thereafter, half of the cultures were supplemented with ghrelin for $24 \mathrm{~h}$, while the other half were not manipulated pharmacologically and used as a control. Before induction of hypoxia and after each experimental phase, part of the neuronal cultures were fixed and processed for detection and quantitative evaluation of the synaptic marker synaptophysin (SPh). The study design is illustrated in Fig. 1.

\section{Dissociated Cell Cultures}

Cell cultures were obtained from Wistar rat pups, from eight plating procedures, five pups (from the same mother) per plating. The animals were anesthetized with isoflurane and decapitated. The brains were removed and placed in RPMI medium (developed at Roswell Park Memorial Institute, hence the
Fig. 1 Scheme of the experimental steps: dissociation of cortical neurons and cell culturing; maturation of the networks for 3-4 weeks; experimental hypoxia for $6 \mathrm{~h}$, followed by $3 \mathrm{~h}$ of recovery at normal oxygen supply, thereafter ghrelin treatment for $24 \mathrm{~h}$. Control cultures were kept for $24 \mathrm{~h}$ in plane medium, not supplemented with ghrelin; immunostaining for detection of synaptophysin at the end of each experimental phase

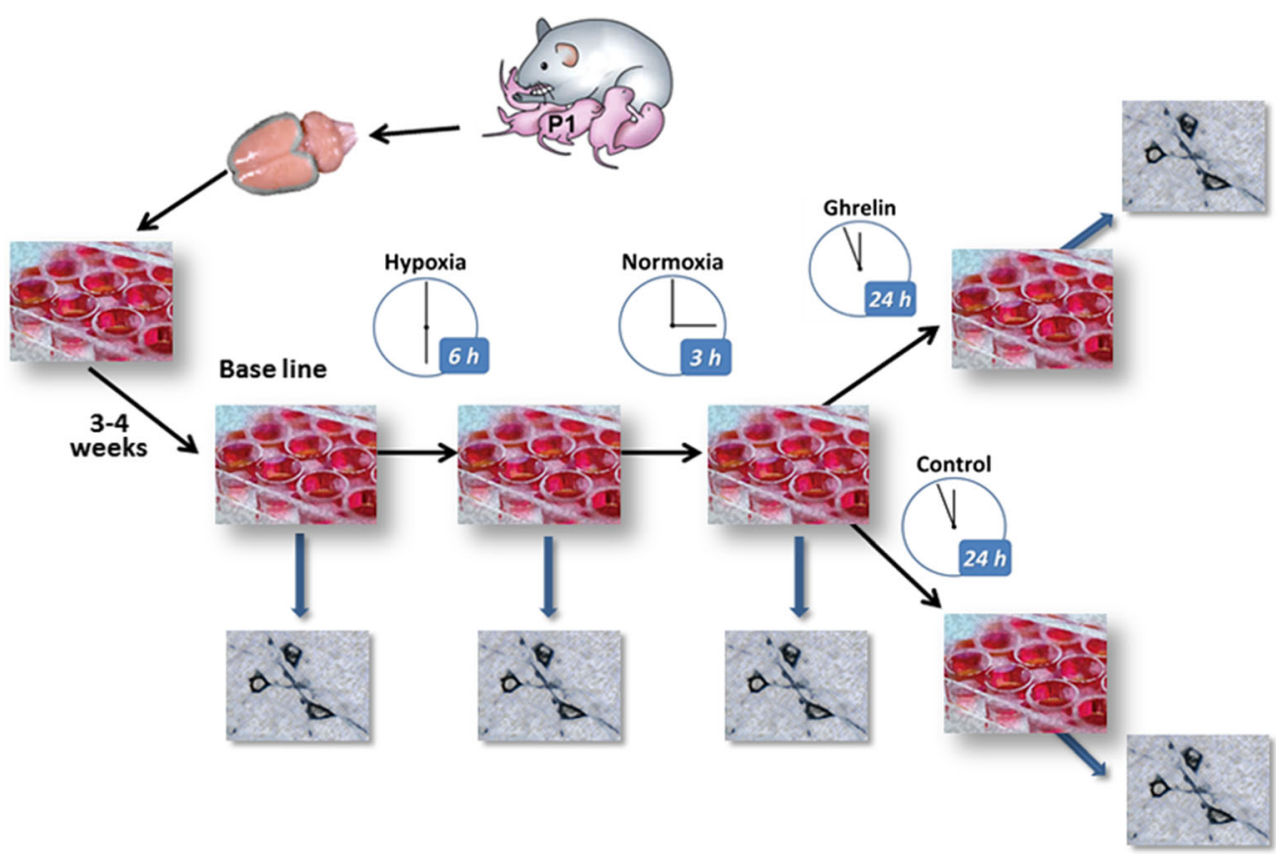


acronym RPMI). After removal of the meninges, cortical cells were dissociated and collected in chemically defined R12 culture medium [35] with trypsin for chemical dissociation. Thereafter, $150 \mu \mathrm{l}$ of soybean trypsin inhibitor and $125 \mu \mathrm{l}$ of DNAse I (20,000 units, Life Technology) were added, followed by trituration for mechanical dissociation of the neurons. The suspension was centrifuged at $1200 \mathrm{rpm}$ for $5 \mathrm{~min}$. Cells were plated on glass coverslips at a density of approximately 3000 cells $/ \mathrm{mm}^{2}$. The glass coverslips were precoated with $20 \mathrm{mg} / \mathrm{ml}$ polyethyleneimine (Fluka, Buchs, Switzerland) to enhance cell adhesion. Cells were allowed to attach for $2 \mathrm{~h}$ at $37{ }^{\circ} \mathrm{C}$ and $5 \% \mathrm{CO}_{2}$ in air and thereafter kept in $\mathrm{R} 12$ medium, optimized with $50 \mathrm{ng} / \mathrm{ml}$ nerve growth factor (Invitrogen, Carlsbad, CA). The medium was serum-free to suppress glial cell proliferation, keeping their concentration lower than $5 \%$ [35]. The medium was renewed every $2-3$ days. Cultures were stored in an incubator under standard conditions of $36^{\circ} \mathrm{C}$, $100 \%$ humidity, and $5 \% \mathrm{CO}_{2}$ for a period of $3-4$ weeks, till the neurons established mature synaptic contacts. All animal experiments were conducted according to Dutch law (as stated in the "Wet op de dierproeven") and approved by the Utrecht Animal Use Committee (DEC).

\section{Induction of Hypoxia and Pharmacological Manipulation}

For induction of hypoxia, the well plates with neuronal cultures were placed under a Plexiglass hood (volume $\sim 5 \mathrm{~L}$ ). Temperature, humidity, and $\mathrm{CO}_{2}$ level of the environment were maintained as in the incubator. Two mass flow controllers were used to mix air and $\mathrm{N}_{2}$ and to produce a total flow of $4.7 \mathrm{~L} / \mathrm{min}$ under the hood. Under physiological conditions, the gas mixture consisted of ambient air with $5 \% \mathrm{CO}_{2}$. For induction of hypoxia, $90 \%$ of the air was replaced by $\mathrm{N}_{2}$ during $6 \mathrm{~h}$, lowering the partial oxygen pressure $\left(\mathrm{pO}_{2}\right)$ in the bath from $\approx 150$ to $\approx 20 \mathrm{~mm} \mathrm{Hg}$. Restoration of normoxia followed thereafter. To obtain an estimate of the course of the oxygen concentration in the culture medium, $\mathrm{pO}_{2}$ was measured using an optical oxygen sensor (PHOSPOR, Ocean Optics), which was inserted into the wells, near the neurons.

Three hours after restoration of normoxia, half of the remaining cultures were supplemented with ghrelin (Abcam, Cambridge, UK) for $24 \mathrm{~h}$, at a final concentration of $1 \mu \mathrm{M}$, as described elsewhere [24, 26, 27, 36, 37]. The other half of the cultures were kept in a plain medium, also for $24 \mathrm{~h}$, and used as control.

\section{Immunohistochemistry}

$\mathrm{SPh}$ staining was done on 55 cultures, equally distributed over the experimental phases (baseline, 6-h hypoxia, 3- $h$ normoxia,

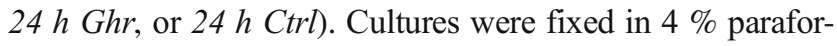
maldehyde in $0.1 \mathrm{M} \mathrm{PBS}, \mathrm{pH} 7.4$, and processed immunocytochemically with the avidin-biotin-horseradish peroxidase
(ABC) method [38] to detect the synaptic marker. Briefly, hydrogen peroxide $(0.3 \%$ in absolute methanol for $30 \mathrm{~min})$ was used to inactivate endogenous peroxidase. Appropriate washes in PBS followed this and subsequent treatments. Incubation in primary antibody mouse anti-SPh IgG (Abcam, Cambridge, UK, dilution 1:1000) lasted for $20 \mathrm{~h}$ at room temperature, followed by $2 \mathrm{~h}$ in biotinilated donkey anti-mouse IgG (1:500; Jackson ImmunoResearch, West) and $1 \mathrm{~h}$ in ABC (1:500; Vector Labs, Burlingame, CA, USA). Following rinsing, peroxidase activity was visualized with $2.4 \%$ SG substrate kit for peroxidase (Vector) in PBS for $5 \mathrm{~min}$, at room temperature. Finally, the cultures were dehydrated in a graded series of alcohols, cleared in xylene, and coverslipped with Entellan (Merck, Darmstadt, Germany).

The immunoreactivity was readily discernible at the light microscopic level by the presence of dark-gray granules of immunoreactive (IR) product. Neuronal structures were considered to be immunopositive when their staining was clearly stronger than that in the background. Negative controls included incubation after antigen-antibody preabsorption with the native antigen, at $4{ }^{\circ} \mathrm{C}$ for $24 \mathrm{~h}$, or replacement of the primary antibody with non-immune serum at the same concentration.

\section{Photomicrograph Production and Data Analysis}

After staining, micrographs were generated at $\times 60$ using a Nikon DS-Fil digital camera linked to a Nikon Eclipse 50i microscope. All digital images were matched for brightness in Adobe Photoshop 7.0. For quantification of the synaptic marker expression, we counted the number of granules of reaction product after $\mathrm{SPh}$ staining. Counting was done blinded to treatment allocation. We obtained estimates of the neuronal size, the number of SPh-IR granules, and the size of the area in which they were counted by means of Nikon NISElements software. To avoid bias due to the small diameter of the neurites and differences in the cell density across the cultures, we restricted this analysis to the area of the perikarya and the initial part of the arborizations.

To guarantee inclusion of neurons of various sizes in our analysis, we defined small-sized (surface of the perikaryon $<100 \mu \mathrm{m}^{2}$ ), medium-sized (cell 100-200 $\mu^{2}$ ), and largesized neurons (cells $>200 \mu \mathrm{m}^{2}$ ) [39-41]. Additionally, we qualitatively graded the overall density of immunostaining of neurons into two categories, high and low, following the procedure described by Ljungdahl et al. [42]. Ten neurons from each category (low and high IR) were examined in each neuronal group (large, medium, or small sized) under each condition (baseline, 6-h hypoxia, 3-h normoxia, $24 \mathrm{~h} \mathrm{Ghr}$, or $24 \mathrm{~h} \mathrm{Ctrl})$. Thus, 60 neurons per condition were evaluated, obtained from 9 to 12 cultures per condition. SPh-IR granule density was calculated and presented as mean \pm standard deviation (SD). Student's $t$ tests and a non-parametric KruskalWallis test with post hoc Tukey HSD were applied to assess 
statistical significance of differences in $\mathrm{SPh}$ expression between the various groups of neurons and conditions. $P$ values $<0.05$ were considered statistically significant.

\section{Results}

Granules of SPh-IR product were localized on the neuronal somata and along the neurites. Their density varied according to experimental condition and according to neuronal size. Typical examples of SPh-IR neurons from each condition group are shown in Fig. 2.

\section{SPh Density According to Experimental Condition}

Hypoxia significantly reduced SPh density $(0.28 \pm 0.06$ granules $\left./ \mu \mathrm{m}^{2}\right)$ compared with baseline $\left(0.34 \pm 0.1\right.$ granules $/ \mu \mathrm{m}^{2}$, $p=0.0002$ ). The posthypoxic values did not return to the baseline levels, neither $3 \mathrm{~h}$ after restoration of normoxia $(0.27 \pm$ 0.07 granules $\left./ \mu \mathrm{m}^{2}\right)$ nor $24 \mathrm{~h}$ later $\left(0.28 \pm 0.15\right.$ granules $\left./ \mu \mathrm{m}^{2}\right)$. However, after ghrelin treatment at $3 \mathrm{~h}, \mathrm{SPh}$ density at $24 \mathrm{~h}$ $\left(0.48 \pm 0.1\right.$ granules $\left./ \mu \mathrm{m}^{2}\right)$ was statistically significant higher than in the control group $\left(0.28 \pm 0.15\right.$ granules $/ \mu \mathrm{m}^{2}, p \ll$ $0.001)$ and at baseline $\left(0.34 \pm 0.1\right.$ granules $\left./ \mu \mathrm{m}^{2}, p \ll 0.001\right)$. The quantitative data are presented in Table 1.

\section{SPh Density According to Neuronal Size}

Synapse density varied according to neuronal size. At baseline, densities were $0.38 \pm 0.08,0.30 \pm 0.06$, and $0.40 \pm 0.12$ granules $/ \mu^{2}$ for large-, medium-, and small-sized neurons. Hypoxia significantly downregulated the number of synaptic contacts of the large neurons $\left(0.29 \pm 0.07\right.$ granules $/ \mu \mathrm{m}^{2}, p=$ $0.0004)$. This was irreversible: the density continued to decrease after restoration of normoxia $(0.24 \pm 0.07$ granules/ $\mu \mathrm{m}^{2}$ after $3 \mathrm{~h}$ and $0.23 \pm 0.14$ granules/ $\mu \mathrm{m}^{2} 24 \mathrm{~h}$ later). Unlike in the large neurons, once reduced by hypoxia, $\mathrm{SPh}$ density of small neurons $\left(0.28 \pm 0.06\right.$ granule $\left./ \mathrm{um}^{2}\right)$ remained unchanged during the posthypoxic periods $\left(0.27 \pm 0.05\right.$ granules $/ \mu \mathrm{m}^{2}$ (3-h restoration) and $24 \mathrm{~h}$ later $\left(0.31 \pm 0.16\right.$ granules $/ \mu \mathrm{m}^{2}$ in $24 \mathrm{~h}$ Ctrl). Synapse density of medium-sized neurons did not show sensitivity to hypoxia. It did not change by exposure to hypoxia $\left(0.27 \pm 0.06\right.$ granules $\left./ \mu \mathrm{m}^{2}\right)$ and remained unchanged after normoxia restoration $\left(0.31 \pm 0.05\right.$ granules $/ \mu \mathrm{m}^{2}$ at $3 \mathrm{~h}$ and $0.28 \pm 0.09$ granules $/ \mu \mathrm{m}^{2} 24 \mathrm{~h}$ later). Ghrelin significantly increased SPh density in the three neuronal subtypes. Treatment effect was largest in the small-sized neurons (Table 2).

\section{Discussion}

In an in vitro model of postanoxic encephalopathy consisting of cultured neurons exposed to temporary severe hypoxia, we
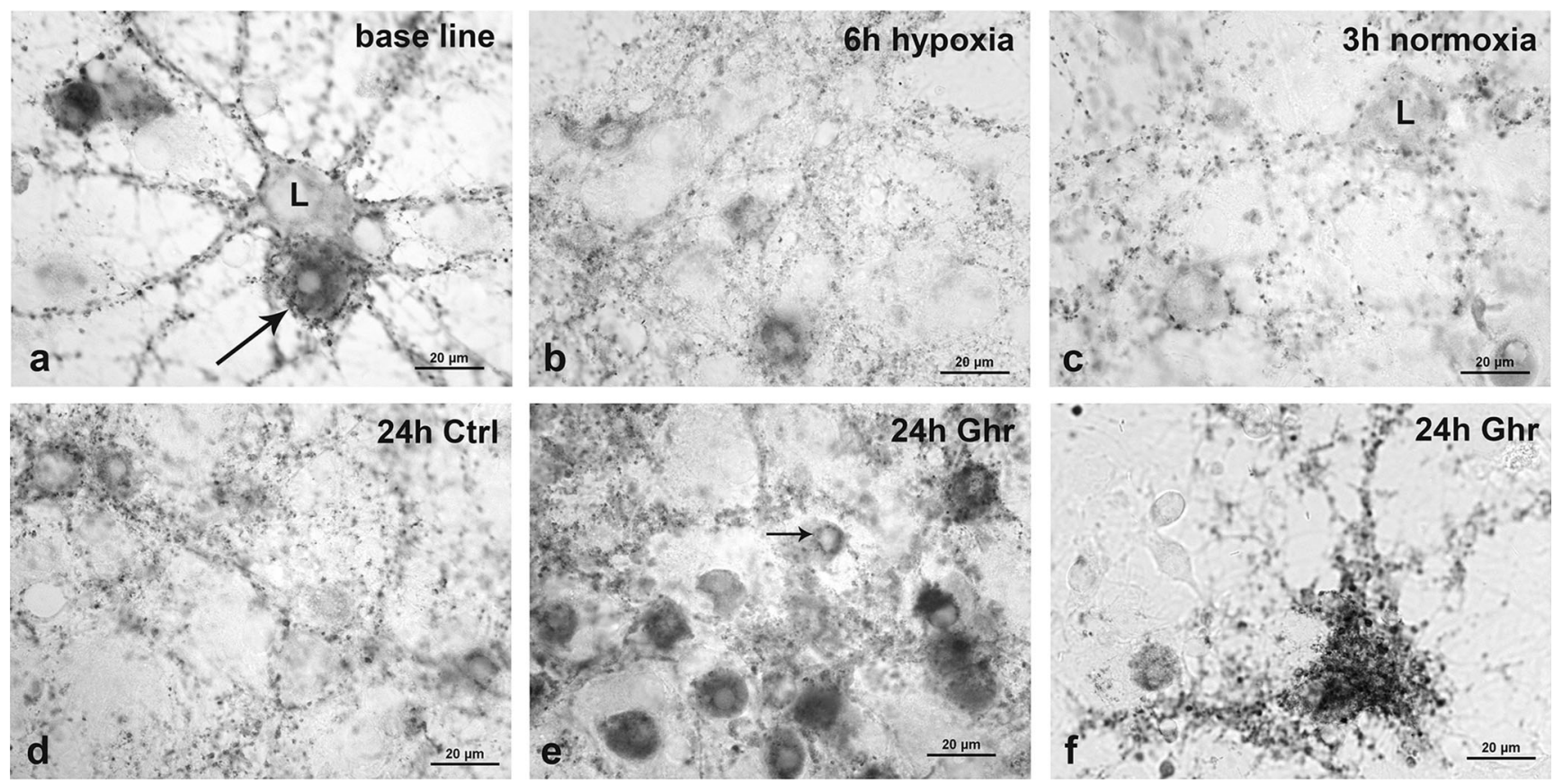

Fig. 2 Microphotographs illustrating SPh-IR in different neuronal subtypes under each experimental condition. a Baseline, large $(L)$ - and small-sized neurons with different $\mathrm{SPh}$ density. The long arrow is pointing at a large neuron with high density of IR granules. b Neurons with low SPh density after $6 \mathrm{~h}$ of hypoxia. $\mathbf{c}$ Neurons of different sizes after 3 -h restoration of oxygen supply. $L$ indicates large-sized neuron. d
Control culture after 24-h recovery in plain medium. e Multiple mediumsized neurons after 24-h recovery in culture supplemented with ghrelin. Most of them show high SPh density. Thin arrow points at a small-sized neuron with low SPh density. f Twenty-four-hour ghrelin treatment, giant pyramidal neuron expressing high SPh density. Scale bars $(\mathbf{a}-\mathbf{f}) 20 \mu \mathrm{m}$ 
Table 1 Density of SPh granules under different experimental conditions

\begin{tabular}{lllll}
\hline Condition & Number of cultures evaluated & Number of neurons evaluated & SPh density $\left(\right.$ granules $\left./ \mu m^{2}\right)$ & SD \\
\hline Baseline & 11 & 60 & 0.34 & \pm 0.10 \\
6-h hypoxia & 9 & 60 & $0.28(* * p=0.0002)$ & $\pm 0.27\left(* * p=6.5 .10^{-5}\right)$ \\
3-h Normoxia & 12 & 60 & $0.28(* p=0.0134)$ & \pm 0.07 \\
24 h Control & 11 & 60 & $0.48\left(* * p=2.10^{-13}\right)$ & \pm 0.15 \\
24 h Ghr & 12 & 60 & \pm 0.10 \\
\hline
\end{tabular}

Asterisks indicate significant change from baseline: $* p<0.05 ; * *<<0.001$

show a significant reduction of synaptic density (from 0.34 to 0.28 granules $/ \mu \mathrm{m}^{2}, p=0.0002$ ). Additionally, we demonstrate that treatment with ghrelin initiates recovery (from 0.28 to 0.48 granules $/ \mu \mathrm{m}^{2}, p \ll 0.001$ ) and leads to complete restoration of the synaptic density in all subtypes of neurons according to their size, with most prominent effect on the mediumsized ones (from 0.28 to 0.48 granules $/ \mu \mathrm{m}^{2}, p \ll 0.001$ ).

Our results are in line with previous in vitro and in vivo studies, showing that hypoxia or ischemia affects synaptic transmission before disruption of ion gradients across the plasma membrane [43-46]. Several causes for presynaptic and postsynaptic ischemic failure have been proposed [17]. The initial disturbances are probably located presynaptically rather than postsynaptically, with impaired transmitter release [17], decreased presynaptic dense projections [47], and isolated loss of presynaptic buttons [48]. Failure of synaptic transmission has been proposed to account for electric silence in the penumbra of a brain infarct [49] and has been associated with lasting network damage in the absence of depolarization in a rat model of cerebral infarction [45]. In addition, perinatal exposure to hypoxia caused early degenerative processes in existing synapses, decline in synaptogenesis, and a more than twofold reduction of synaptic density in all cortical layers of the rat, as shown by SPh immunodetection [50]. Cerebral ischemia induces interaction of the postsynaptic density (PSD-95) with neuronal nitric oxide synthase (nNOS), thereby leading to nitric oxide (NO) overproduction and neural injury [51]. Disruption of this coupling enhances neurite growth and dendritic spine formation and thus improves stroke outcome by promoting regenerative processes [52].
Further research on the effect of hypoxia on postsynaptic density and dendritic spine distribution would be clinically relevant.

Previously demonstrated selective ischemic damage affected striatum, hippocampal pyramidal neurons, cerebellar Purkinje cells, and the neocortical pyramidal cells [53-55] with relatively strong downregulation of synapse density [48]. In the cortex, we also observed selective vulnerability: large- and small-sized neurons were more susceptible to synaptic damage than medium-sized neurons. In general, neuronal morphology correlates with certain neuronal functions, and as the fundamental work of McConnell indicated, the large-sized neurons convey afferent sensory signals from subcortical regions and efferent signaling to subcortical areas, while the small-sized neurons are involved in efferent intracortical transmission [56]. Therefore, we could speculate that the primary sensory and motor systems are more vulnerable to ischemic damage. Such morphological evidence for selective vulnerability of the primary sensory and motor system was demonstrated in a model of asphyxic cardiac arrest in newborn piglets [57] and confirmed electrophysiologically in rodents [58]. Causes of selective vulnerability are still unclear; however, it is possible to be related to specific oxidative metabolism [57].

Previously, we reported that AG accelerates synaptogenesis and synaptic activity under healthy conditions [23, 24]. Supposed working mechanisms for stimulating synapse growth or recovery include activation of several signaling pathways. By activating GHSR1, ghrelin increases $\mathrm{Ca}^{+2}$ influx $[59,60]$ and intracellular $\mathrm{Ca}^{+2}$ mobilization [61] which

Table 2 SPh density in different neuronal types under all experimental conditions

\begin{tabular}{|c|c|c|c|c|c|}
\hline \multirow[t]{2}{*}{ Neuronal subtype } & \multicolumn{5}{|c|}{ Mean SPh density (granules $/ \mu \mathrm{m}^{2}$ ) $\pm \mathrm{SD}$} \\
\hline & Baseline & 6-h hypoxia & 3-h normoxia & $24 \mathrm{~h} \mathrm{Ctrl}$ & $24 \mathrm{~h} \mathrm{Ghr}$ \\
\hline Large & $0.38 \pm 0.08$ & $0.29 \pm 0.07\left(* * p=4.10^{-4}\right)$ & $0.24 \pm 0.07\left(* * p=1.3 .10^{-6}\right)$ & $0.23 \pm 0.14\left(* * p=2.8 .10^{-4}\right)$ & $0.45 \pm 0.08(* p=0.01)$ \\
\hline Medium & $0.30 \pm 0.06$ & $0.27 \pm 0.06$ & $0.31 \pm 0.05$ & $0.28 \pm 0.09$ & $0.48 \pm 0.09\left(* * p=1.1 .10^{-8}\right)$ \\
\hline Small & $0.40 \pm 0.12$ & $0.28 \pm 0.06\left(* * p=3.8 .10^{-4}\right)$ & $0.27 \pm 0.05\left(* * p=1.4 .10^{-4}\right)$ & $0.31 \pm 0.16(* p=0.04)$ & $0.52 \pm 0.12(* p=0.003)$ \\
\hline
\end{tabular}

Asterisks indicate significant change from baseline: $* p<0.05 ; * *<0.001$ 
results in synaptic gene nuclear factor expression [62]. Furthermore, as an excitatory neurotransmitter, ghrelin stimulates synaptic activity, which triggers additional synthesis and aggregation of neurotransmitter receptors [63] and other types of synaptic signaling molecules like agrin [64]. In the cerebral cortex, agrin is associated with excitatory but not inhibitory synapses [65]. It stimulates formation and stabilization of dendritic filopodia and consequently promotes synaptogenesis [66]. Additionally, agrin induces a switch from gap junctionmediated communication to synaptic transmission [67]. Considering that gap junctions respond to hypoxia-ischemia by excessive channel opening which spreads the injury [68, 69], it is reasonable to hypothesize a role for ghrelin in the restriction of ischemic injury and upregulation of the synaptic assembly via agrin-mediated mechanism.

Beneficial effects of ghrelin have been observed in animal models of acute ischemic stroke. In a rat model, administration of ghrelin reduces infarct volume and cell death [70] by preventing apoptosis and improving mitochondrial function [71]. Thus, in addition to the stimulating effect on the synaptic survival, ghrelin further ameliorates cellular respiration and network recovery from severe hypoxia.

We used cultured neuronal networks as a model system to study the effects of temporary severe hypoxia. An important advantage of this model system is that, unlike acute brain slices, cultures can stay alive for several days up to months. In addition, many neurons and synapses in a network can be studied simultaneously. A possible limitation is the lack of normal brain architecture, as present in acute brain slices. However, the focus of this study was on general synaptic damage and recovery, which does not require a specific brain structure. The system has been used before, but with a focus on anoxia-induced neuronal death instead of synaptic function [72].

In conclusion, the present observations support the hypothesis that exogenous ghrelin has a robust impact on the recovery of cortical synapses after hypoxia. It raises the possibility that ghrelin or its analogs may have a therapeutic potential for treatment of postanoxic encephalopathy.

Acknowledgments We thank Dr. GC Hassink and Bettie Klomphaar for their assistance in cell culturing.

Open Access This article is distributed under the terms of the Creative Commons Attribution 4.0 International License (http:// creativecommons.org/licenses/by/4.0/), which permits unrestricted use, distribution, and reproduction in any medium, provided you give appropriate credit to the original author(s) and the source, provide a link to the Creative Commons license, and indicate if changes were made.

\section{References}

1. Zandbergen EG et al (1998) Systematic review of early prediction of poor outcome in anoxic-ischaemic coma. Lancet 352(9143): $1808-1812$

2. (1986) Randomized clinical study of thiopental loading in comatose survivors of cardiac arrest. Brain Resuscitation Clinical Trial I Study Group. N Engl J Med 314(7):397-403

3. Group, B.R.C.T.I.S. (1991) A randomized clinical study of a calcium-entry blocker (lidoflazine) in the treatment of comatose survivors of cardiac arrest. Brain Resuscitation Clinical Trial II Study Group. N Engl J Med 324(18):1225-1231

4. Roine RO et al (1990) Nimodipine after resuscitation from out-ofhospital ventricular fibrillation. A placebo-controlled, double-blind, randomized trial. JAMA 264(24):3171-3177

5. Thel MC et al (1997) Randomised trial of magnesium in in-hospital cardiac arrest. Duke Internal Medicine Housestaff. Lancet 350(9087):1272-1276

6. Longstreth WT Jr et al (2002) Randomized clinical trial of magnesium, diazepam, or both after out-of-hospital cardiac arrest. Neurology 59(4):506-514

7. Group, H.a.C.A.S (2002) Mild therapeutic hypothermia to improve the neurologic outcome after cardiac arrest. N Engl J Med 346(8): 549-556

8. Bernard SA et al (2002) Treatment of comatose survivors of out-ofhospital cardiac arrest with induced hypothermia. N Engl J Med 346(8):557-563

9. Nielsen $\mathrm{N}$ et al (2013) Targeted temperature management at 33 degrees $\mathrm{C}$ versus 36 degrees $\mathrm{C}$ after cardiac arrest. N Engl J Med 369(23):2197-2206

10. Andresen $\mathrm{M}$ et al (2015) Therapeutic hypothermia for acute brain injuries. Scand J Trauma Resusc Emerg Med 23:42

11. Ginsberg MD (2008) Neuroprotection for ischemic stroke: past, present and future. Neuropharmacology 55(3):363-389

12. Symon L et al (1977) The concepts of thresholds of ischaemia in relation to brain structure and function. J Clin Pathol Suppl (R Coll Pathol) 11:149-154

13. Gutierrez LG et al (2010) CT and MR in non-neonatal hypoxicischemic encephalopathy: radiological findings with pathophysiological correlations. Neuroradiology 52(11):949-976

14. Mlynash $\mathrm{M}$ et al (2010) Temporal and spatial profile of brain diffusion-weighted MRI after cardiac arrest. Stroke 41(8):16651672

15. Tsai YW et al (2013) Post ischemia intermittent hypoxia induces hippocampal neurogenesis and synaptic alterations and alleviates long-term memory impairment. J Cereb Blood Flow Metab 33(5): 764-773

16. Sun MK et al (2008) Poststroke neuronal rescue and synaptogenesis mediated in vivo by protein kinase $\mathrm{C}$ in adult brains. Proc Natl Acad Sci U S A 105(36):13620-13625

17. Hofmeijer J, van Putten MJ (2012) Ischemic cerebral damage: an appraisal of synaptic failure. Stroke 43(2):607-615

18. Hofmeijer J et al (2014) Mild hypoxia affects synaptic connectivity in cultured neuronal networks. Brain Res 1557:180-189

19. Ghosh A, Carnahan J, Greenberg ME (1994) Requirement for BDNF in activity-dependent survival of cortical neurons. Science 263(5153):1618-1623

20. Butz M, Worgotter F, van Ooyen A (2009) Activity-dependent structural plasticity. Brain Res Rev 60(2):287-305

21. Tjepkema-Cloostermans MC et al (2015) Electroencephalogram predicts outcome in patients with postanoxic coma during mild therapeutic hypothermia. Crit Care Med 43(1):159-167

22. Hofmeijer $\mathbf{J}$ et al. (2015) Early EEG contributes to multimodal outcome prediction of postanoxic coma. Neurology 
23. Stoyanova II, le Feber J, Rutten WL (2013) Ghrelin stimulates synaptic formation in cultured cortical networks in a dosedependent manner. Regul Pept 186:43-48

24. Stoyanova II, le Feber J (2014) Ghrelin accelerates synapse formation and activity development in cultured cortical networks. BMC Neurosci 15:49

25. Kojima M et al (1999) Ghrelin is a growth-hormone-releasing acylated peptide from stomach. Nature 402(6762):656-660

26. Diano $\mathrm{S}$ et al (2006) Ghrelin controls hippocampal spine synapse density and memory performance. Nat Neurosci 9(3):381-388

27. Cowley MA et al (2003) The distribution and mechanism of action of ghrelin in the CNS demonstrates a novel hypothalamic circuit regulating energy homeostasis. Neuron 37(4):649-661

28. Stoyanova II, Wiertz RW, Rutten WL (2009) Time-dependent changes in ghrelin-immunoreactivity in dissociated neuronal cultures of the newborn rat neocortex. Regul Pept 158(1-3):86-90

29. Salmeron C et al (2015) Roles of leptin and ghrelin in adipogenesis and lipid metabolism of rainbow trout adipocytes in vitro. Comp Biochem Physiol A Mol Integr Physiol 188:40-48

30. Hosoda H et al (2000) Ghrelin and des-acyl ghrelin: two major forms of rat ghrelin peptide in gastrointestinal tissue. Biochem Biophys Res Commun 279(3):909-913

31. van der Lely AJ (2009) Ghrelin and new metabolic frontiers. Horm Res 71(Suppl 1):129-133

32. Kang $\mathrm{S}$ et al (2015) Central acylated ghrelin improves memory function and hippocampal AMPK activation and partly reverses the impairment of energy and glucose metabolism in rats infused with beta-amyloid. Peptides 71:84-93

33. Li E et al (2013) Ghrelin directly stimulates adult hippocampal neurogenesis: implications for learning and memory. Endocr $\mathrm{J}$ 60(6):781-789

34. Kent BA et al (2015) The orexigenic hormone acyl-ghrelin increases adult hippocampal neurogenesis and enhances pattern separation. Psychoneuroendocrinology 51:431-439

35. Romijn HJ, van Huizen F, Wolters PS (1984) Towards an improved serum-free, chemically defined medium for long-term culturing of cerebral cortex tissue. Neurosci Biobehav Rev 8(3):301-334

36. Johansson I et al (2008) Proliferative and protective effects of growth hormone secretagogues on adult rat hippocampal progenitor cells. Endocrinology 149(5):2191-2199

37. Cecarini V et al. (2015) Effects of ghrelin on the proteolytic pathways of Alzheimer's disease neuronal cells. Mol Neurobiol

38. Hsu SM, Raine L, Fanger H (1981) Use of avidin-biotin-peroxidase complex $(\mathrm{ABC})$ in immunoperoxidase techniques: a comparison between $\mathrm{ABC}$ and unlabeled antibody (PAP) procedures. J Histochem Cytochem 29(4):577-580

39. Gittins R, Harrison PJ (2004) Neuronal density, size and shape in the human anterior cingulate cortex: a comparison of Nissl and NeuN staining. Brain Res Bull 63(2):155-160

40. Gittins R, Harrison PJ (2004) A quantitative morphometric study of the human anterior cingulate cortex. Brain Res 1013(2):212-222

41. Unger JW, Lange W (1992) NADPH-diaphorase-positive cell populations in the human amygdala and temporal cortex: neuroanatomy, peptidergic characteristics and aspects of aging and Alzheimer's disease. Acta Neuropathol 83(6):636-646

42. Ljungdahl A, Hokfelt T, Nilsson G (1978) Distribution of substance P-like immunoreactivity in the central nervous system of the rat-I. Cell bodies and nerve terminals. Neuroscience 3(10):861-943

43. Leblond J, Krnjevic K (1989) Hypoxic changes in hippocampal neurons. J Neurophysiol 62(1):1-14

44. Fujiwara $\mathrm{N}$ et al (1987) Effects of hypoxia on rat hippocampal neurones in vitro. J Physiol 384:131-151

45. Bolay $\mathrm{H}$ et al (2002) Persistent defect in transmitter release and synapsin phosphorylation in cerebral cortex after transient moderate ischemic injury. Stroke 33(5):1369-1375
46. Sun MK, Xu H, Alkon DL (2002) Pharmacological protection of synaptic function, spatial learning, and memory from transient hypoxia in rats. J Pharmacol Exp Ther 300(2):408-416

47. Stepanov SS et al (1998) An ultrastructural study into the effect of global transient cerebral ischaemia on the synaptic population of the cerebellar cortex in rats. Resuscitation 39(1-2):99106

48. Horner CH, Davies HA, Stewart MG (1998) Hippocampal synaptic density and glutamate immunoreactivity following transient cerebral ischaemia in the chick. Eur J Neurosci 10(12): 3913-3917

49. Astrup J, Siesjo BK, Symon L (1981) Thresholds in cerebral ischemia - the ischemic penumbra. Stroke 12(6):723-725

50. Otellin VA, Khozhai LI, Shishko TT (2014) Reactions of the interneuronal synapses of rat brain to hypoxia during the early postnatal period. Morfologiia 145(1):7-12

51. Zhou L et al (2010) Treatment of cerebral ischemia by disrupting ischemia-induced interaction of nNOS with PSD-95. Nat Med 16(12):1439-1443

52. Luo CX et al (2014) Interaction of nNOS with PSD-95 negatively controls regenerative repair after stroke. J Neurosci 34(40):1353513548

53. Cervos-Navarro J, Diemer NH (1991) Selective vulnerability in brain hypoxia. Crit Rev Neurobiol 6(3):149-182

54. Bendel $\mathrm{O}$ et al (2005) Reappearance of hippocampal CA1 neurons after ischemia is associated with recovery of learning and memory. J Cereb Blood Flow Metab 25(12):15861595

55. Schwartz PH et al (1992) A rat model of severe neonatal hypoxicischemic brain injury. Stroke 23(4):539-546

56. McConnell SK (1991) The generation of neuronal diversity in the central nervous system. Annu Rev Neurosci 14:269300

57. Martin LJ et al (1997) Primary sensory and forebrain motor systems in the newborn brain are preferentially damaged by hypoxia-ischemia. J Comp Neurol 377(2):262-285

58. Wu D et al (2012) Short- and long-latency somatosensory neuronal responses reveal selective brain injury and effect of hypothermia in global hypoxic ischemia. J Neurophysiol 107(4): 1164-1171

59. Hosoda H, Kojima M, Kangawa K (2006) Biological, physiological, and pharmacological aspects of ghrelin. J Pharmacol Sci 100(5):398-410

60. Dominguez B, Felix R, Monjaraz E (2007) Ghrelin and GHRP-6 enhance electrical and secretory activity in GC somatotropes. Biochem Biophys Res Commun 358(1):59-65

61. Herrington J, Hille B (1994) Growth hormone-releasing hexapeptide elevates intracellular calcium in rat somatotropes by two mechanisms. Endocrinology 135(3):1100-1108

62. Lyons MR, West AE (2011) Mechanisms of specificity in neuronal activity-regulated gene transcription. Prog Neurobiol 94(3):259 295

63. Broadie K, Bate M (1993) Activity-dependent development of the neuromuscular synapse during Drosophila embryogenesis. Neuron 11(4):607-619

64. Nastuk MA, Fallon JR (1993) Agrin and the molecular choreography of synapse formation. Trends Neurosci 16(2):72-76

65. Ksiazek I et al (2007) Synapse loss in cortex of agrin-deficient mice after genetic rescue of perinatal death. J Neurosci 27(27):71837195

66. McCroskery S et al (2009) Transmembrane agrin regulates dendritic filopodia and synapse formation in mature hippocampal neuron cultures. Neuroscience 163(1):168-179

67. Martin AO, Alonso G, Guerineau NC (2005) Agrin mediates a rapid switch from electrical coupling to chemical neurotransmission during synaptogenesis. J Cell Biol 169(3):503-514 
68. Talhouk RS et al (2008) Gap junctional intercellular communication in hypoxia-ischemia-induced neuronal injury. Prog Neurobiol 84(1):57-76

69. Frantseva MV, Kokarovtseva L, Perez Velazquez JL (2002) Ischemia-induced brain damage depends on specific gapjunctional coupling. J Cereb Blood Flow Metab 22(4):453462
70. Chung $\mathrm{H}$ et al (2007) Ghrelin inhibits apoptosis in hypothalamic neuronal cells during oxygen-glucose deprivation. Endocrinology 148(1):148-159

71. Andrews ZB (2011) The extra-hypothalamic actions of ghrelin on neuronal function. Trends Neurosci 34(1):31-40

72. Rothman SM (1983) Synaptic activity mediates death of hypoxic neurons. Science 220(4596):536-537 